\title{
THREE-DIMENSIONAL COMPUTATIONS OF FREE-BURNING ARCS AND THEIR SURROUNDINGS
}

\author{
Won-Ho LeE ${ }^{a}$, Jong-Chul LeE ${ }^{b, *}$ \\ ${ }^{a}$ Graduate School of Automotive Engineering, Gangneung-Wonju National University, Wonju, Republic of Korea \\ ${ }^{b}$ School of Mechanical and Automotive Engineering, Gangneung-Wonju National University, Wonju, Republic of \\ Korea \\ * jclee01@gwnu.ac.kr
}

\begin{abstract}
This paper is concerned with developing a capability to model free-burning high-intensity argon arcs and enhancing the accuracy of numerical results according to three-dimensional calculations. It was found that the computed temperatures along the axis between the cathode tip and the anode surface show good agreement with two different measured data. Although the LTE model can reasonably predict the overall arc voltage for free-burning arcs, it fails to account accurately what happens at the regions near electrodes.
\end{abstract}

Keywords: free-burning arc, thermal plasma, computational fluid dynamics, LTE model.

\section{Introduction}

A free-burning arc where the work piece acts as an anode is an idealized representative of the DC arcs and has been used for industrial processes such as cutting, welding, spraying, coating, material heating and melting, lighting, current interruption and more recently for waste disposal, production of fine particles and thermal plasma vapor deposition [1]. Because a scientific understanding of the behavior of the arc itself and its electrode regions is very important for improving industrial applications, numerous theoretical and experimental papers have been published in the last decade to understand the thermal behavior of arcs and their electrodes [2]. Because the flow patterns inside the free-burning arc system must be of the 3-dimensional feature, 2-dimensional modeling cannot help in predicting the 3 -dimensional flow and heat transfer within the systems.

In the present work, we developed a threedimensional model of free-burning argon arcs with a commercial computational fluid dynamics (CFD) package, ANSYS-CFX, which has a flexibility to modify the governing equations for taking into account radiation transport, electrical power input and the electromagnetic driving forces. The comparison of the arc temperatures with two different measured data is used to judge the validation of the present computations.

\section{Numerical Methods}

Two different free-burning arc systems for computations are shown in Figure 1, where one is called as HF model [3] and the other SF model [4]. For the arc modeling involved complicated interactions between the flow and electromagnetic fields, we modified the Navier-Stokes equations to take into account the radiation transport, the electrical power input

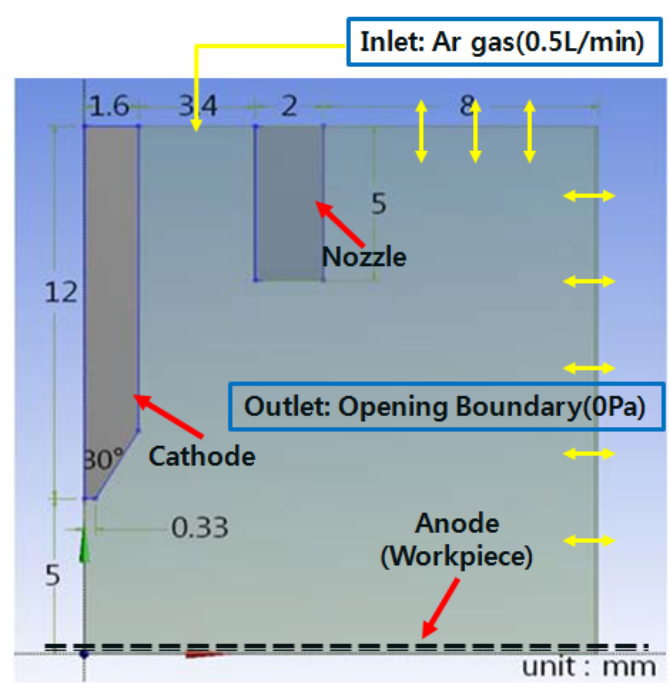

(a) HF model

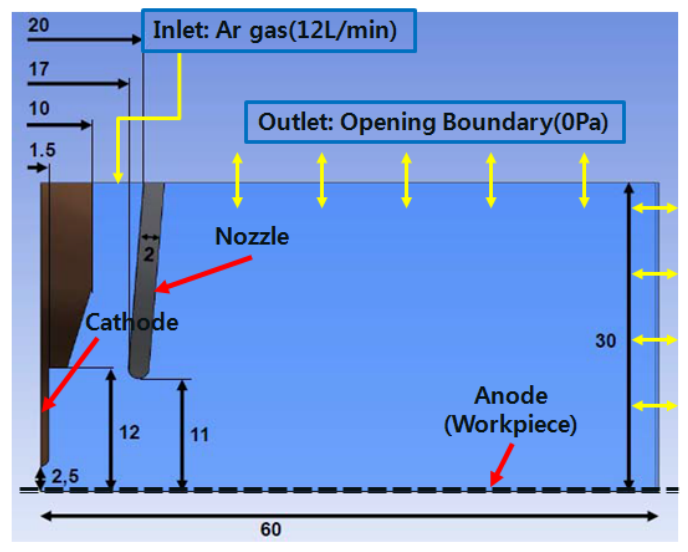

(b) SF model

Figure 1. Schematic diagram with boundary condition of two different free-burning arc systems; (a)HF model; (b) SF model 


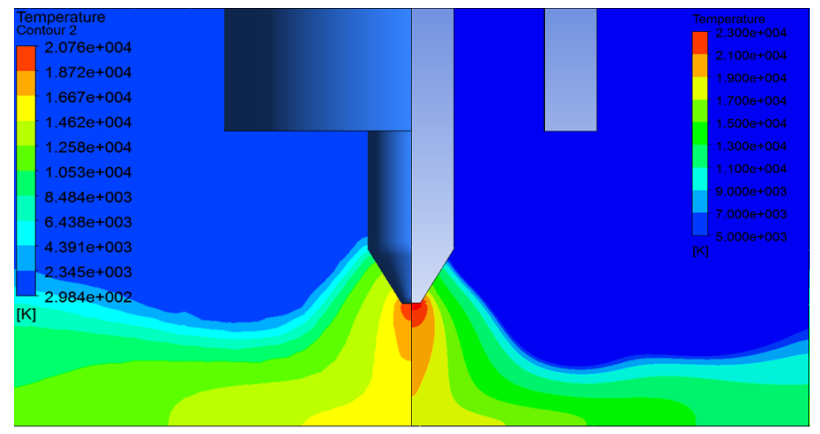

(a) HF model

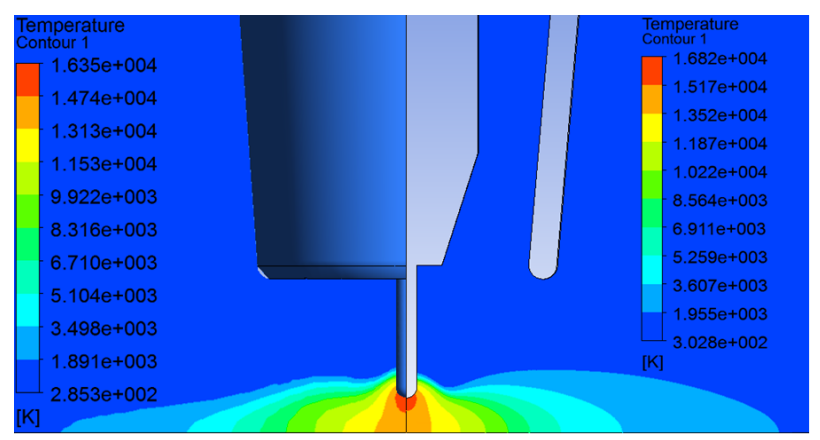

(b) SF model

Figure 2. Comparison of temperature distributions in the arc column for 200A by 3-D and 2-D computations; (a)HF model; (b) SF model.

and the electromagnetic driving forces with the relevant Maxwell equations. The stationary conservation equations can be written in the generalized form suggested by Patankar as shown in [5, 6]. The thermodynamic and the transport properties, such as density, enthalpy, constant-pressure specific heat, viscosity, electrical and thermal conductivities, and optically thin radiation losses, required for this calculation strongly depend on the temperature and the pressure. The data adopted in this study have been taken from 7]. Radiation transport in high pressure arcs is an important energy transport mechanism. In the present work, the net radiation loss in the energy equation is computed using a semi-empirical radiation transport model [8]. The radiation transport model requires the use of net emission coefficients of argon, which are given by Menart et al. [9].

\section{Results and Discussion}

Figure 2 shows the computed temperature distributions of two different free-burning arc systems, HF model and SF model, to compare the results between 2-D and 3-D simulations. Each figure consists of two results; 3-D computational result is in left-hand side and 2-D computational result is in right-hand side at the centerline. Both 2-D and 3-D results show that the temperature near the cathode is higher than that near the anode due to the arc constriction near the cathode by the magnetic pinch effect. The argon entrained from the flow inlet is heated strongly and accelerates rapidly, resulting in the formation of the cathode jet. The temperature in the arc region presents the typical bell shape of high-intensity arcs. It is because the plasma jet approaches the anode, the axial velocity decreases rapidly and turns the flow direction toward the r-direction near the anode. It can be seen that the computed temperature profiles by 2-D and 3-D are in good agreement each other at both HF and SF models. It can be also seen that the temperature of HF model is higher than that of $\mathrm{SF}$ model because the gap distance of HF model is

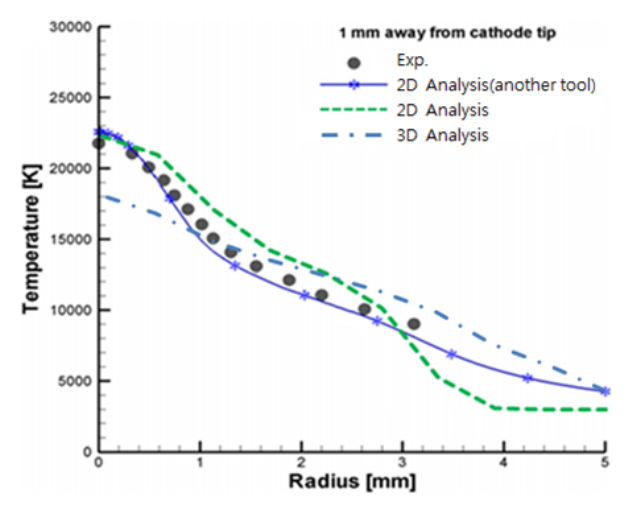

(a) $1 \mathrm{~mm}$

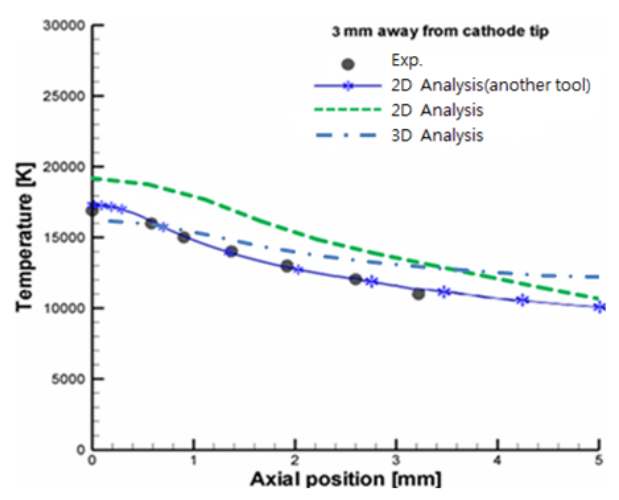

(b) $3 \mathrm{~mm}$

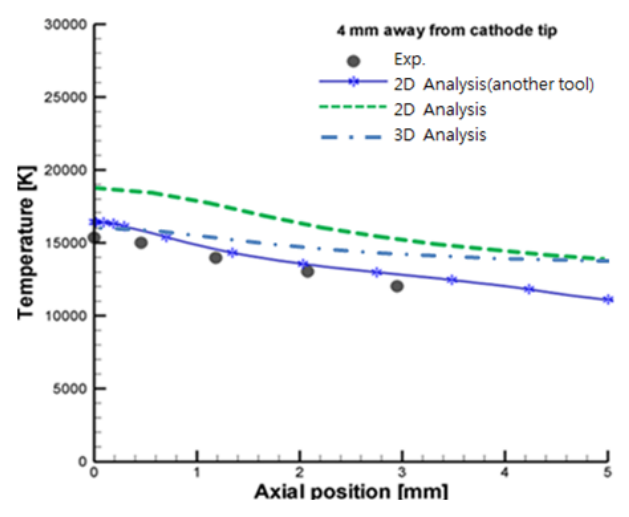

(c) $4 \mathrm{~mm}$

Figure 3. Temperature profiles of HF model away from the cathode;(a)1mm; (b) $3 \mathrm{~mm}$; (c) $4 \mathrm{mml}$. 


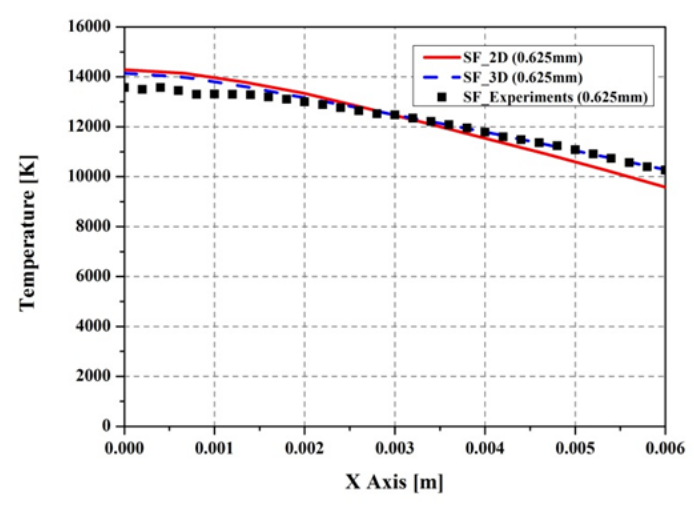

(a) $0.625 \mathrm{~mm}$

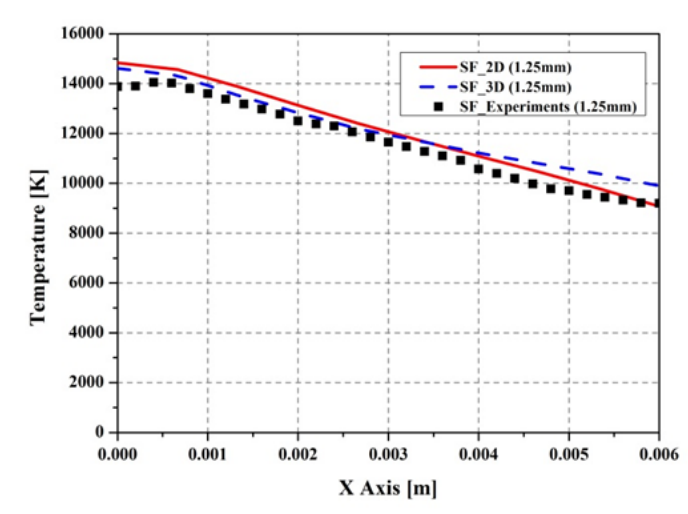

(b) $1.25 \mathrm{~mm}$

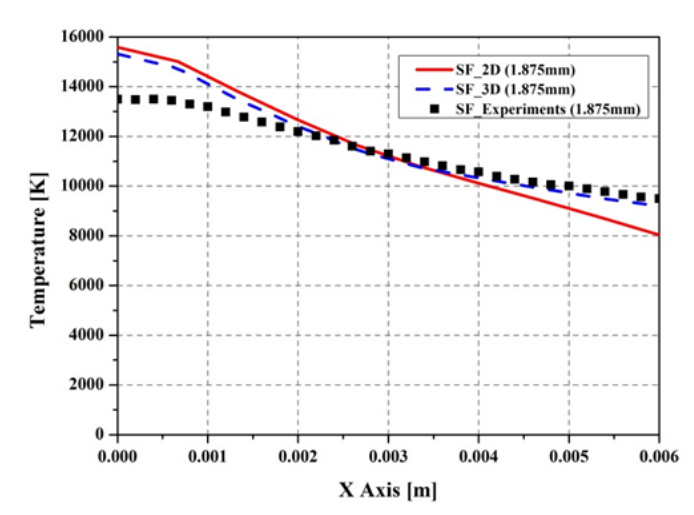

(c) $1.875 \mathrm{~mm}$

Figure 4. Temperature profiles of SF model away from the cathode;(a)0.625 $\mathrm{mm}$; (b) $1.25 \mathrm{~mm}$; (c)1.875mml.

longer than that of SF model. We thus conclude that our 3-D model produces satisfactory results for known cases. Detailed comparisons between the calculated and measured temperatures at different radial position are given in Figs. 3 and 4 Figure 3 shows the radial variation of the temperature of $\mathrm{HF}$ model at $1 \mathrm{~mm}$, $3 \mathrm{~mm}, 4 \mathrm{~mm}$ away from the cathode. The results show reasonable agreement with those measured by Haddad and Farmer [3]. The 3-D predicted arc temperature on the axis is slightly lower than the measurement near the cathode but still within the experimental uncertainty. Figure 4 shows the radial variation of the temperature of SF model at $0.65 \mathrm{~mm}, 1.25 \mathrm{~mm}$, $1.875 \mathrm{~mm}$ away from the cathode. As shown in Fig. 3 of $\mathrm{HF}$ model, the computed results of SF model are also in agreement with those measured by [10]. At both HF and SF models, it can be seen that the predicted temperature profiles by $3-\mathrm{D}$ are in better agreement with the measured data than 2-D. However, it fails to account accurately what happens at the near electrode region, especially near the cathode. An accurate solution near electrodes has to be based on non-LTE model to ensure current continuation in the low temperature region.

\section{Conclusion}

In this study, we have concerned with developing a capability to model free-burning high-intensity argon arcs and enhancing the accuracy of numerical results according to three-dimensional calculations. It was found that the predicted temperature profiles with $3-\mathrm{D}$ are in better agreement with the measured data than 2-D. However, it fails to account accurately what happens at the near electrode region, especially near the cathode.

\section{Acknowledgements}

This research was supported by Basic Science Research Program through the National Research Foundation of Korea(NRF) funded by the Ministry of Education, Science and Technology (NRF-2015R1D1A1A09058389).

\section{References}

[1] M. T. C. Fang, J. L. Zhang, and J. D. Yan. On the use of langmuir probes for the diagnosis of atmospheric thermal plasmas. IEEE Trans. on Plasma Sci., 33(4):1431-1441, 2005.

[2] J. J. Gleizes, J. Gonzalez, and J. Freton. Thermal plasmas modeling. J. Phys. D: Appl. Phys, 38(9):R153-R183, 2005.

[3] G. N. Haddad and A. J. D. Farmer. Temperature determinations in a free-burning arc: I. experimental techniques and results in argon. J. Phys. D: Appl. Phys., 17(6):1189-1196, 1984.

[4] M. Schnick, U. Fussel, M. Hertel, S. Rose, M. Haessler, A. Spille-Kohoff, and A. B. Murphy. Numerical investigations of the influence of metal vapour in gma welding. Welding in the World, 55(11):114-120, 2011.

[5] S. V. Patankar. Numerical Heat Transfer and Fluid Flow. McGraw-Hill, New Yorkt, 1980.

[6] W. H. Lee, Y. J. Kim, and J. C. Lee. Temperature prediction in a free-burning arc and electrodes for nanostructured materials and systems. J. Nanosci. Nanotechnol, 15(11):8446-8450, 2015.

[7] A. B. Murphy. A comparison of treatments of diffusion in thermal plasmas. J. Phys. D: Appl. Phys., 29:1922, 1996.

[8] P. Kovitya and J. J. Lowke. Two-dimensional analysis of free-burning arcs in argon. J. Phys. D: Appl. Phys., 18:53, 1985. 
[9] J Menart, J. Heberlein, and E. Pfender. Theoretical radiative emission results for argon/copper thermal plasmas. Plasma Chem. Plasma Process, 16:245s, 1995.

[10] A. Spille-Kohoff. Arc welding: From process simulation to structural mechanics, Part 1: Process simulation with ANSYS CFX. 4th European Automotive Simulation Conference, Munich, Germany, 6-7 July, 2009. 